\title{
Planar metal plasmon waveguides: frequency-dependent dispersion, propagation, localization, and loss beyond the free electron model
}

\author{
J. A. Dionne,* L. A. Sweatlock, and H. A. Atwater \\ California Institute of Technology, Watson Laboratories of Applied Physics, MC 128-95, Pasadena, California 91125, USA \\ A. Polman \\ California Institute of Technology, Watson Laboratories of Applied Physics, MC 128-95, Pasadena, California 91125, USA \\ and The Center for Nanophotonics, FOM-Institute AMOLF, Kruislaan 407, 1098 SJ Amsterdam, The Netherlands \\ (Received 15 February 2005; revised manuscript received 13 May 2005; published 2 August 2005)
}

\begin{abstract}
A numerical analysis of surface plasmon dispersion, propagation, and localization on smooth lossy films is presented. Particular attention is given to determining wavelength-dependent behavior of thin $\mathrm{Ag}$ slab waveguides embedded in a symmetric $\mathrm{SiO}_{2}$ environment. Rather than considering $\mathrm{Ag}$ as a damped free electron gas, the metal is defined by the experimentally determined optical constants of Johnson and Christy and Palik. As in free electron gas models, analytic dispersion results indicate a splitting of plasmon modescorresponding to symmetric and antisymmetric field distributions-as film thickness is decreased below $50 \mathrm{~nm}$. However, unlike free electron gas models, the surface plasmon wave vector remains finite at resonance with the antisymmetric-field plasmon converging to a pure photon mode for very thin films. In addition, allowed excitation modes are found to exist between the bound and radiative branches of the dispersion curve. The propagation characteristics of all modes are determined, and for thin films (depending upon electric field symmetry), propagation distances range from microns to centimeters in the near infrared. Propagation distances are correlated with both the field decay (skin depth) and energy density distribution in the metal and surrounding dielectric. While the energy density of most long-range surface plasmons exhibits a broad spatial extent with limited confinement in the waveguide, it is found that high-field confinement does not necessarily limit propagation. In fact, enhanced propagation is observed for silver films at ultraviolet wavelengths despite strong field localization in the metal. The surface plasmon characteristics described in this paper provide a numerical springboard for engineering nanoscale metal plasmon waveguides, and the results may provide a new avenue for integrated optoelectronic applications.
\end{abstract}

DOI: 10.1103/PhysRevB.72.075405

PACS number(s): 73.20.Mf, 73.40.Rw, 42.82.Et

\section{INTRODUCTION}

Since Ritchie ${ }^{1}$ first described the plasma losses of electrons incident on a metal film, collective electronic motion has received considerable attention. In 1967 Kliewer and Fuchs $^{2}$ determined the dispersion characteristics of thin-film surface plasmons (SPs), considering the conduction electrons as a free electron gas (FEG). Subsequently, Economou ${ }^{3}$ extended the theory to multilayer metallodielectric systems, using a FEG dielectric function but including retardation effects.

In 1985, Burke and Stegeman ${ }^{4}$ reported on damped surface plasma oscillations in a metal film, solving the thin-film dispersion relations at a given frequency (free space wavelength $\lambda=633 \mathrm{~nm}$ ) and determining wavelength-dependent attenuation by modifying the FEG dielectric function with a Drude damping term. For thin films excited at $\lambda=1 \mu \mathrm{m}$, multicentimenter propagation was found, providing the first hint that surface plasmons might have profound applications in the design of optoelectronic integrated circuits. At present, surface plasmon metal optics is an area of active research, promising potential for coupling, confining, and guiding light in the nanometer scale regime.

In the broadest sense, a surface plasmon is an electron density fluctuation bounded at the interface between a conductor and an insulator. Nonradiative in nature, these elec- tromagnetic surface oscillations (termed Fano modes ${ }^{5}$ ) cannot couple to photons without resort to a prism, grating or spatially localized scattering center to resolve the momentum mismatch. Experimentally, surface plasmons are excited and detected either through near-field techniques (see, for instance, Refs. 6-9) or through far-field microscopy and spectroscopy measurements, including electron energy loss spectroscopy (EELS). ${ }^{10-12}$ Propagation lengths on the order of $10-100 \mu \mathrm{m}$ have been observed in the visible, ${ }^{6,13}$ and losses of less than $10 \mathrm{~dB} / \mathrm{cm}$ have been reported in $\mathrm{Ag}$ wires excited in the infrared. ${ }^{14}$

To date, experimental dispersion and attenuation results have not contradicted the theoretical predictions of planar slab surface plasmons. However, many analytical models lack a quantitative consideration of SP properties that arise when the metal is described by experimentally determined optical constants rather than a damped free electron gas. And, if models do employ empirical optical constants, ${ }^{4,15-18}$ attenuation characteristics are determined for a given frequency rather than throughout a broad spectral range. Moreover, few works have explicitly determined the spatial decay of the plasmon electric field and energy density into the metal and dielectric as a function of film thickness. From both a fundamental and an applied perspective, quantitative knowledge of such wavelength-dependent SP characteristics seems essential. 
This paper discusses both thick and thin film surface plasmon behavior arising from the experimentally determined optical constants of silver as adopted from Johnson and Christy $^{19}$ (JC) and Palik. ${ }^{20,21}$ Particular attention is given to mapping the dispersion relations, determining wavelengthdependent propagation and electric field skin depth, and characterizing energy density decay into the relevant passive media.

Section II provides an overview of the optical characterization of silver, summarizing the techniques of Johnson and Christy and Palik and detailing the specific dielectric functions used throughout this paper. In subsequent sections, direct comparison between SP behavior using both sets of optical constants is made, highlighting the discrepancies between them. Section III reviews thick-film plasmon behavior, deriving the dispersion relation without the common assumption that the magnitude of the real component of the dielectric constant exceeds the imaginary component (see, for instance, Ref. 22). This formalism introduces plasmon modes in the classical "plasmon bandgap" between bound and radiative SP modes. Section IV outlines thin-film plasmon properties, including results on wavelength-dependent dispersion, propagation, and localization of all modes. Attention is given to describing the physical significance of the numerical techniques (based on EELS theory and a NelderMead minimization routine) used in solving the dispersion relations. The paper concludes with a general discussion of long-range surface plasmons and their applications to integrated photonics.

\section{Ag OPTICAL CHARACTERIZATION}

The free electron gas model was introduced by Drude to describe general electron transport in metals. In this theory, the dielectric function takes the form

$$
\varepsilon(\omega)=1-\frac{\omega_{p}^{2}}{\omega(\omega+i \gamma)},
$$

where $\omega_{p}$ defines the bulk plasma frequency of the material and $\gamma$ denotes the relaxation rate. Separating $\varepsilon(\omega)$ into real and imaginary components and assuming $\omega \gg \gamma$, this expression simplifies to

$$
\varepsilon(\omega)=1-\left(\frac{\omega_{p}}{\omega}\right)^{2} .
$$

While this form of the dielectric function is commonly quoted as an adequate optical characterization of metallic films (see, for instance, Refs. 2, 3, 23, and 24, which use this form to determine surface plasmon dispersion), its validity is limited only to near-infrared frequencies. A more accurate description of SP behavior throughout the electromagnetic spectrum requires use of empirically determined optical constants.

Bulk Ag is most often described by the refractive index $(n+i \cdot k)$ data sets of Johnson and Christy and from the Palik Handbook of Optical Constants of Solids. ${ }^{20}$ The optical constants of Johnson and Christy were determined through measurements of reflection and transmission at normal incidence

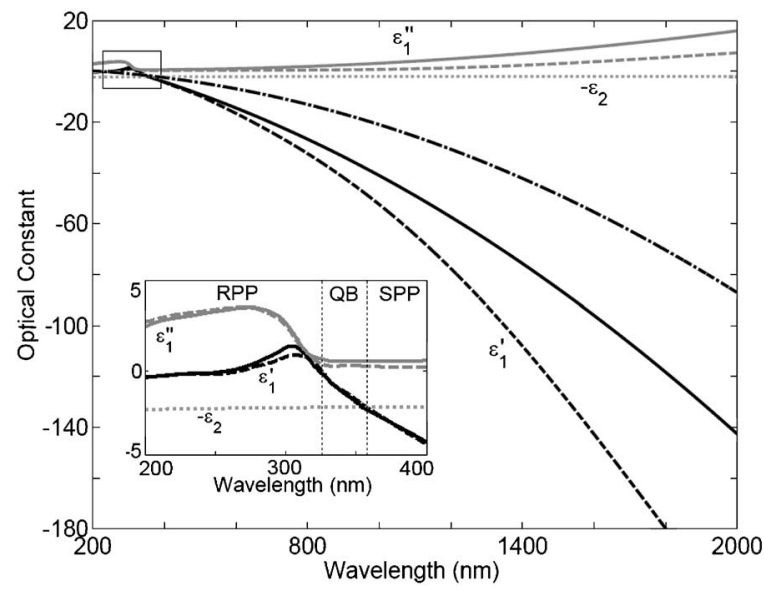

FIG. 1. The real and imaginary components of the Ag dielectric function $\left(\varepsilon_{1}=\varepsilon_{1}{ }^{\prime}+i \varepsilon_{1}{ }^{\prime \prime}\right)$ using a free-electron model (dash-dot line type), data from Johnson and Christy (dashed), and the Palik Handbook (solid). For reference, the polynomial fit through $\mathrm{Palik}^{\prime} \mathrm{SiO}_{2}$ data is also included (dotted, plotted as $-\varepsilon_{2}$ ). The inset shows an enlarged view of the region of anomalous dispersion for the Johnson and Christy and Palik data sets.

and transmission of $p$-polarized light at $60^{\circ}$. In contrast, the optical constants compiled by Palik (including the works of Leveque $^{25}$ from 200 to $360 \mathrm{~nm}$, Winsemius ${ }^{26}$ from 360 to $2000 \mathrm{~nm}$, and Dold and $\mathrm{Mecke}^{27}$ from 1265 to $2000 \mathrm{~nm}$ ) were obtained via reflectance measurements using synchrotron radiation $^{25}$ and polarimetric measurements. ${ }^{26,27}$

Figure 1 shows the dielectric function $\varepsilon_{1}(\omega)=\varepsilon_{1}{ }^{\prime}(\omega)$ $+i \cdot \varepsilon_{1}{ }^{\prime \prime}(\omega)$ of $\mathrm{Ag}$ as derived from the data of both Johnson and Christy and Palik. For reference, Fig. 1 also includes the FEG dielectric function of Eq. (2) and the dielectric constant of the embedding dielectric, $\mathrm{SiO}_{2}\left(\varepsilon_{2}\right.$, plotted as $\left.-\varepsilon_{2}\right)$, adopted from Palik. To describe the dielectric function continuously between 200 and $2000 \mathrm{~nm}$, the raw empirical data was fit using a cubic spline within the region of anomalous dispersion (i.e., the region where $\operatorname{Re}\left[\varepsilon_{1}(\omega)\right]$ decreases with increasing $\omega$ (decreasing wavelength); see Fig. 1 inset) and polynomial fits elsewhere. All fits were designed to be continuous and smooth throughout the wavelength range $200 \mathrm{~nm} \leqslant \lambda \leqslant 2000 \mathrm{~nm}$ and agree with the experimental data within $5 \%$. At shorter wavelengths $(\lambda<400 \mathrm{~nm})$, the agreement is within $0.01 \%$. Details are given in the Appendix.

As seen, the JC and Palik data sets are in fair accord for wavelengths between 200 and $600 \mathrm{~nm}$. However, significant differences are observed between 600 and $2000 \mathrm{~nm}$, with $\varepsilon_{1}{ }^{\prime}$ of Johnson and Christy falling off faster with wavelength and $\varepsilon_{1}{ }^{\prime \prime}$ growing more slowly than the corresponding data of Palik. Given this inconsistency, and given the general use of both data sets to describe bulk silver, surface plasmon properties in this paper were separately derived considering each data set.

\section{THICK Ag FILMS: DECOUPLED SURFACE PLASMONS}

Though the properties of surface plasmons bound to a single metal-dielectric interface are well developed in scien- 


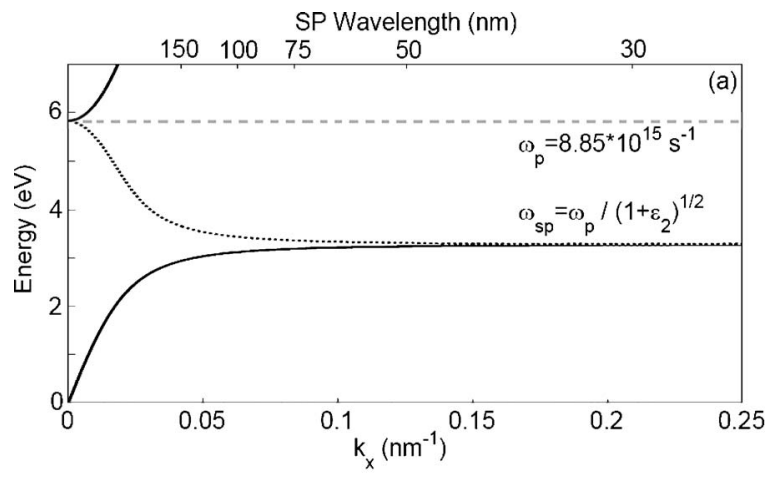

$$
\begin{gathered}
E_{y}^{\text {metal }}=E_{y}^{\text {dielectric }}=0, \\
E_{z}^{\text {metal }}=E_{0}\left(\frac{-k_{x}}{k_{z 1}}\right) e^{i\left(k_{x} x-k_{z 1}|z|-\omega t\right)}, \\
E_{z}^{\text {dielectric }}=E_{0}\left(\frac{-\varepsilon_{1} k_{x}}{\varepsilon_{2} k_{z 1}}\right) e^{i\left(k_{x} x-k_{z 2}|z|-\omega t\right)} .
\end{gathered}
$$

Demanding continuity of the tangential $\mathbf{E}$ and normal D fields at the interface yields the typical surface plasmon dispersion relations defined by ${ }^{22}$

$$
k_{x}=\frac{\omega}{c} \sqrt{\frac{\varepsilon_{1} \varepsilon_{2}}{\varepsilon_{1}+\varepsilon_{2}}} \text { and } k_{z 1,2}^{2}=\varepsilon_{1,2}\left(\frac{\omega}{c}\right)^{2}-k_{x}^{2} .
$$

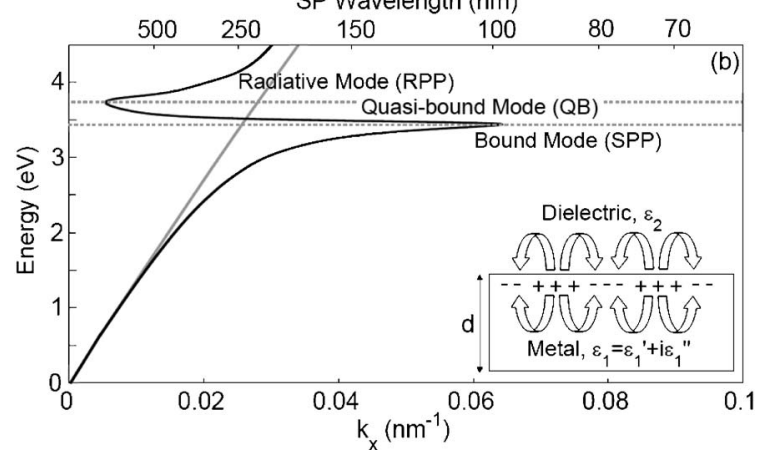

FIG. 2. (a) Surface plasmon dispersion relation for the $\mathrm{Ag} / \mathrm{SiO}_{2}$ geometry computed using a free electron gas dispersion model. Note the existence of allowed modes (solid) for frequencies below $\omega_{\text {SP }}$ and above $\omega_{p}$, in contrast to the forbidden (i.e., purely imaginary) modes between these frequencies (dotted). (b): Bound (SPP), radiative (RPP), and quasibound (QB) surface plasmon dispersion relation for the $\mathrm{Ag} / \mathrm{SiO}_{2}$ geometry computed using the optical constants of Johnson and Christy. Unlike the free electron dispersion of panel (a), modes are allowed throughout the entire frequency range shown. The $\mathrm{SiO}_{2}$ light line (gray) is also included for reference.

tific literature, few studies provide a coherent numerical comparison of dispersion, propagation, field skin depth, and energy density using empirically determined optical constants. Here we present such data for an $\mathrm{Ag} / \mathrm{SiO}_{2}$ surface plasmon excitation over the full wavelength range of Fig. 1.

Properly considered, surface plasmons are the quanta of collective plasma oscillations localized at the interface between a metal and a dielectric. Provided the thickness of the metal film exceeds the plasmon skin depth, oscillations at each metal-dielectric interface are decoupled, and independent surface plasmon modes at each metal-dielectric interface are sustained. A cross section of the geometry is shown as an inset in Fig. 2; the metal is contained in the half space $z>0$ with the metallodielectric interface located at $z=0$. Wave propagation is along the $x$ direction.

Assuming a perpendicularly polarized electric field incident on the structure, the surface plasmon electric field takes the form

$$
E(x, z, t) \sim E_{0} e^{i\left(k_{x} x-k_{z}|z|-\omega t\right)}
$$

with components given by

$$
E_{x}^{\text {metal }}=E_{0} e^{i\left(k_{x} x-k_{z 1}|z|-\omega t\right)}, \quad E_{x}^{\text {dielectric }}=E_{0} e^{i\left(k_{x} x-k_{z 2}|z|-\omega t\right)},
$$

Provided $\varepsilon_{1}{ }^{\prime \prime}<\left|\varepsilon_{1}{ }^{\prime}\right|$-a condition satisfied in $\mathrm{Ag}$ for $\lambda$ $>328 \mathrm{~nm}$ in the Johnson and Christy data set and $\lambda$ $>331 \mathrm{~nm}$ in the Palik set-the in-plane wave vector can be written as $k_{x}=k_{x}{ }^{\prime}+i k_{x}{ }^{\prime \prime}$, with

$$
k_{x}{ }^{\prime}=\frac{\omega}{c} \sqrt{\frac{\varepsilon_{1}{ }^{\prime} \varepsilon_{2}}{\varepsilon_{1}{ }^{\prime}+\varepsilon_{2}}} \text { and } k_{x}^{\prime \prime}=\frac{\omega}{c}\left(\frac{\varepsilon_{1}{ }^{\prime} \varepsilon_{2}}{\varepsilon_{1}{ }^{\prime}+\varepsilon_{2}}\right)^{3 / 2}\left(\frac{\varepsilon_{1}{ }^{\prime \prime}}{2 \varepsilon_{1}}\right) \text {. }
$$

Figure 2(a) illustrates the dispersion characteristics for this mode, plotted using the dielectric function of a free electron gas with $\omega_{p}=8.85 \times 10^{15} \mathrm{~s}^{-1}{ }^{28}$ For energies below $3.3 \mathrm{eV}$, the typical bound surface plasmon-polariton (SPP) mode is observed, asymptoting at short wave vectors to the light line and at large wave vectors to the surface plasmon resonant frequency $\omega_{\mathrm{SP}}$ (defined by the wavelength where $\varepsilon_{1}{ }^{\prime}=-\varepsilon_{2}$ ). Above $5.8 \mathrm{eV}$, the onset of the radiative plasmon-polariton (RPP) mode can be seen. For energies between the SPP and RPP modes, the plasmon wave vector is purely imaginary (represented as a dotted line in the figure), indicating that modes in this regime are forbidden. Historically, this region between $\omega_{\mathrm{SP}}$ and $\omega_{p}$ is referred to as the plasmon bandgap.

In contrast to the free electron behavior, dispersion arising from use of Johnson and Christy optical constants is plotted in Fig. 2(b). Though not shown, dispersion is nearly identical using the optical constants of Palik. For reference, the dispersion curve for the $\mathrm{SiO}_{2}$ light line $\left(k_{x}=\varepsilon_{2}{ }^{1 / 2} \omega / \mathrm{c}\right)$ is also included. Below $3.5 \mathrm{eV}$ the SPP mode is observed, approaching the light line at short wave vectors but terminating at a finite wave vector on resonance $\left(k_{x}=0.065 \mathrm{~nm}^{-1}\right.$ at $\left.\omega_{\text {SP }}\right) .{ }^{29}$ As seen, the fairly large SPP wave vectors (and hence small SPP wavelengths) achieved near resonance compete with the largely reduced group velocity in this frequency range. Above $3.8 \mathrm{eV}$ the RPP is observed, corresponding to wavelengths satisfying the relation $\varepsilon_{1}{ }^{\prime \prime}>\left|\varepsilon_{1}{ }^{\prime}\right|$ (i.e., $\lambda$ $<328 \mathrm{~nm}$ ). For energies between the SPP and RPP modes, $k_{x}$ is determined by Eq. (4) and what we term quasibound (QB) modes appear to exist. Unlike the imaginary modes of the FEG dispersion, the modes plotted here have mathematically real components and hence are not a priori forbidden.

Quasibound modes may provide an opportunity to study negative phase velocities in naturally occurring materials. Nevertheless, the seemingly infinite group velocity marking the transition regimes between SPP/QB modes and QB/RPP modes is - at first sight - a disconcerting feature. In normal 


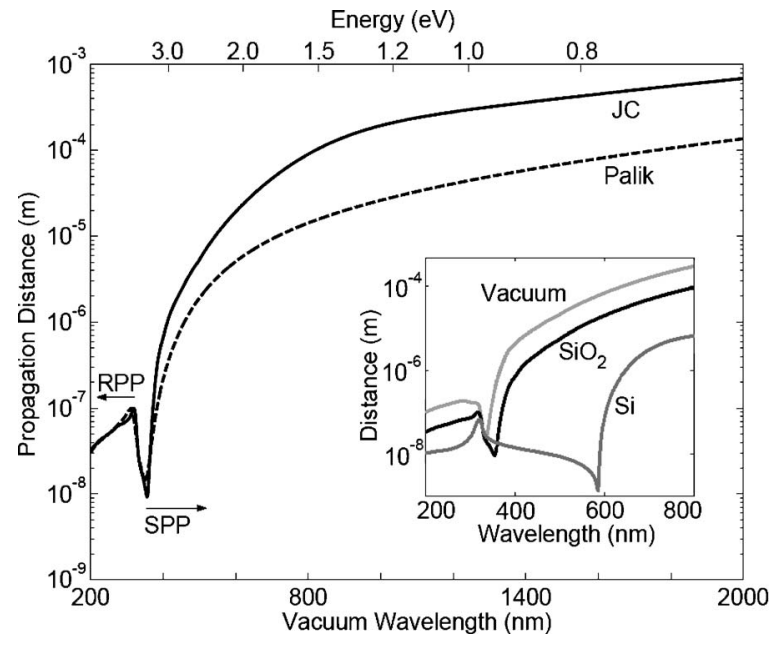

FIG. 3. Surface plasmon propagation length for the $\mathrm{Ag} / \mathrm{SiO}_{2}$ geometry calculated using the optical constants of Johnson and Christy (solid) and Palik (dotted). A vanishing propagation length occurs at the surface plasmon resonance, located at $\lambda=355 \mathrm{~nm}$ $(358 \mathrm{~nm}$ ) for Palik (JC). The local maximum at $\lambda \sim 320 \mathrm{~nm}$ coincides with the transition between QB and RPP modes. Inset: Comparison of SP propagation for $\mathrm{Ag} / \mathrm{Air}, \mathrm{Ag} / \mathrm{SiO}_{2}$, and $\mathrm{Ag} / \mathrm{Si}$ geometries plotted about the SP resonance.

dispersive media, the group velocity is defined by the relation $v_{g}=d \omega / d k$. However, in regions of anomalous dispersion this linearization does not apply, and the propagation velocity of the wave packet must be modified to account for amplitude damping and wave profile deformation. ${ }^{30} \mathrm{~A}$ detailed discussion of QB mode properties (including group velocity and dispersion) is deferred to another paper. However, these modes may exhibit significant physical effects on the time scale of short-lifetime plasmon processes, including plasmon decoherence by electron-hole pair generation and electron-phonon coupling.

Figure 3 illustrates the propagation distance for an $\mathrm{Ag} / \mathrm{SiO}_{2}$ interface plasmon as a function of wavelength for both the Johnson and Christy and Palik dielectric data sets. The surface plasmon intensity decreases as $\exp \left(2 \operatorname{Re}\left[i k_{x} x\right]\right)$ so that the propagation length is given by $L=\left|2 \operatorname{Re}\left[i k_{x}\right]\right|^{-1}$. As seen, at the important telecommunications wavelength of $1550 \mathrm{~nm}$, propagation distance approaches $\sim 400 \mu \mathrm{m}$ using the Johnson and Christy dielectric function data set and $\sim 70 \mu \mathrm{m}$ using Palik; at shorter wavelengths, both curves converge toward nanometer-scale propagation at the surface plasmon resonance. Thus, although large surface plasmon wave vectors (and therefore short plasmon wavelengths) can be achieved near resonance, these attributes are often at the expense of propagation length.

Still, Fig. 3 does reveal the presence of a local maximum in propagation existing close to the plasmon resonant frequency. Indicating the transition between the QB and RPP modes, the neighborhood about this maximum corresponds to the region of anomalous dispersion in the silver dielectric constant. Accordingly, the relative magnitude of this maximum can be controlled by altering the refractive index of the surrounding dielectric.

To illustrate the effects of varying the dielectric optical constant, the inset of Fig. 3 plots propagation lengths for

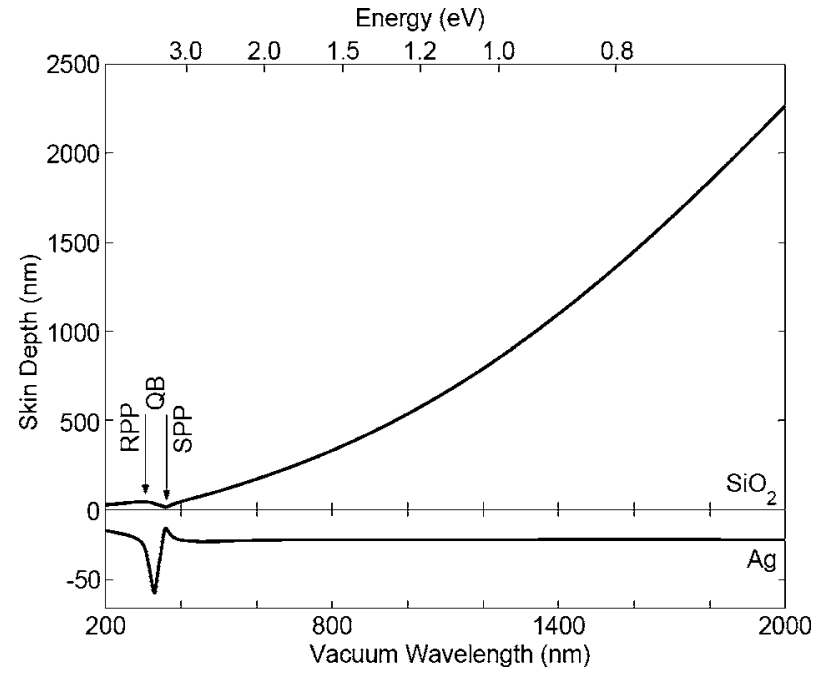

FIG. 4. Surface plasmon electric field penetration depth into Ag and $\mathrm{SiO}_{2}$, computed using the dielectric data of Johnson and Christy. The local penetration maximum at shorter wavelengths corresponds to the transition between the quasibound and radiative surface plasmon modes.

$\mathrm{Ag} / \mathrm{Air}, \mathrm{Ag} / \mathrm{SiO}_{2}$, and $\mathrm{Ag} / \mathrm{Si}$ (Ref. 31) interface plasmons over the spectral range of $200-500 \mathrm{~nm}$. By using materials of varying refractive indices, the surface plasmon resonant frequency can be tuned through a broad spectral range. As a result, the energetic location of the maxima and minima in the propagation distance plot can be controlled. Alternatively, at a given frequency, the surface plasmon wavelength (and hence damping) can be tuned by the dielectric constant. Such results suggest that by altering the optical properties of the embedding dielectric (i.e., with an electro-optic material), propagation might be dynamically switched under optical pumping. However, significant shifts for thick films require refractive index differentials of order $\Delta n=0.1^{32}$ : at a free space wavelength of $\lambda=1550 \mathrm{~nm}$, such index contrast can lead to a $6 \mu \mathrm{m}$ change in propagation length.

The inset also reveals that for a given free-space wavelength, the longest propagation lengths are achieved for insulating materials with the smallest dielectric constant. However, combining data as in Figs. 2(b) and 3 for various dielectrics (data not shown), we find that the optimal combination of small plasmon wavelength and low damping is observed for a dielectric with the largest refractive index.

Figure 4 plots the surface plasmon electric field skin depth (1/e decay length) in both $\mathrm{Ag}$ and $\mathrm{SiO}_{2}$ as a function of wavelength. Note that in this graph, decay into the metal is plotted below $z=0$. For clarity, the figure only includes skin depth computed using the JC data set. As expected, the SPP skin depth into the dielectric increases with increasing wavelength, reaching values of $1.3 \mu \mathrm{m}(1.1 \mu \mathrm{m})$ in $\mathrm{SiO}_{2}$ at $\lambda_{\text {vac }}=1550 \mathrm{~nm}$ for the JC (Palik) data sets, respectively. In contrast, the SPP skin depth into the metal remains roughly constant at $\sim 20 \mathrm{~nm}(25 \mathrm{~nm})$ for wavelengths beyond the plasmon resonance. At resonance, field penetration in the metal reaches a minimum in both the metal and dielectric (with $z \sim 15 \mathrm{~nm}$ for both $\mathrm{Ag}$ and $\mathrm{SiO}_{2}$ ). For even shorter wavelengths, field penetration reaches a maximum in the 

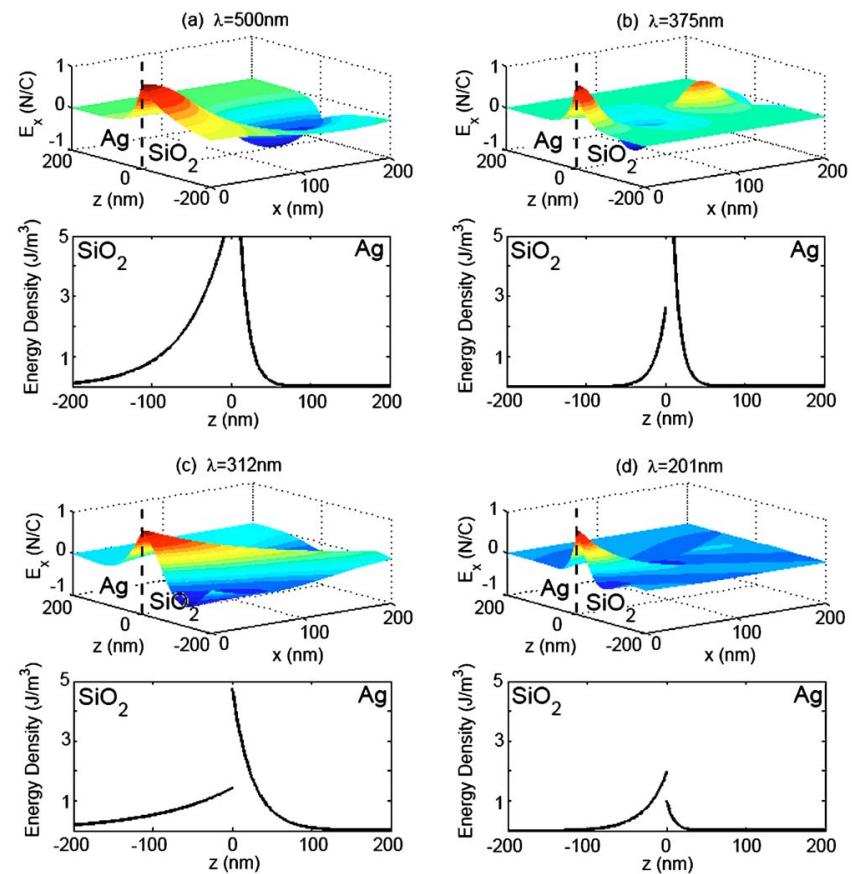

FIG. 5. (Color online) Spatial distribution of the surface plasmon tangential field and corresponding electric energy density in the metal $(z>0)$ and dielectric $(z<0)$ for wavelengths characteristic of the surface plasmon-polariton modes, (a) $\lambda=500 \mathrm{~nm}$, (b) $\lambda$ $=375 \mathrm{~nm}$, and the radiative plasmon-polariton modes, (c) $\lambda$ $=312 \mathrm{~nm}$, (d) $\lambda=201 \mathrm{~nm}$.

metal and a local maximum in $\mathrm{SiO}_{2}\left(z_{\mathrm{Ag}} \sim 60 \mathrm{~nm}, z_{\mathrm{SiO}_{2}}\right.$ $\sim 40 \mathrm{~nm}$ ) for $\lambda \sim 330 \mathrm{~nm}$ - a wavelength regime corresponding to anomalous $\mathrm{Ag}$ dispersion and marking the onset of the radiative plasmon polariton.

This observation raises an interesting distinction between the system resonances corresponding to the extrema in the plot of propagation length versus wavelength (i.e., the local maximum and the global minimum in Fig. 3). At one system resonance, corresponding to anomalous dispersion, propagation is slightly enhanced despite high field confinement within the metal. However, at the surface plasmon resonance, propagation approaches single nanometer scales despite minimal energy density within the metal. The latter result is explained by the vanishing SPP excitation group velocity near the plasmon resonance. Still, the contradiction to the common localization versus loss heuristic is evident: field localization within the metal does not necessarily result in increased loss.

Figure 5 illustrates the tangential field $\left(E_{x}\right)$ distribution and electric energy density decay into both the metal and the dielectric as a function of distance from the metallodielectric interface for wavelengths characteristic of the SPP and RPP modes. Only results obtained using the Johnson and Christy data sets are shown; the behavior is qualitatively similar considering dielectric function data from Palik. Outside the waveguide, the energy density is defined in the usual way, with

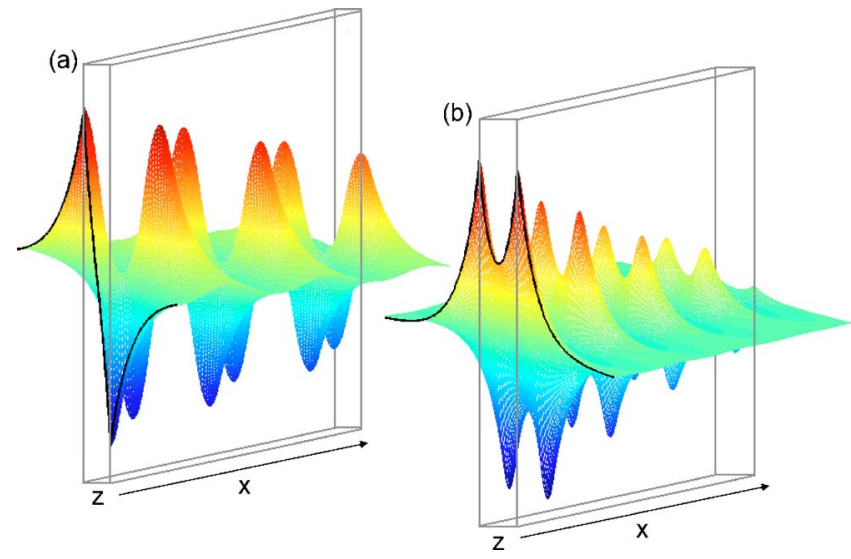

FIG. 6. (Color online) Characteristic electric field profile for the metal slab waveguide with thickness $z=d$. (a) The higher energy, antisymmetric field mode $(L+)$; (b) the lower energy, symmetric field mode $(L-)$. The propagation direction $x$ is indicated by arrows.

$$
u_{\mathrm{SiO}_{2}}=\frac{1}{2} \vec{E} \cdot \vec{D}^{*}=\frac{1}{2} \varepsilon_{2} \vec{E} \cdot \vec{E}^{*}
$$

while within the waveguide, the energy density is derived from Poynting's theorem in linear, lossy dispersive media: ${ }^{33}$

$$
u_{\mathrm{Ag}}=\frac{1}{2} \operatorname{Re}\left[\frac{d\left(\omega \varepsilon_{1}\right)}{d \omega}\right] \vec{E} \cdot \vec{E}^{*} .
$$

Figures 5(a) and 5(b) show the SPP mode and energy density at $2.48 \mathrm{eV}\left(\lambda_{\mathrm{vac}}=500 \mathrm{~nm}\right)$ and $3.31 \mathrm{eV}\left(\lambda_{\mathrm{vac}}=375 \mathrm{~nm}\right)$, respectively. Calculations are made for an incident field normalized to $1 N / C$. As the free-space wavelength is decreased toward the surface plasmon resonance, the energy density becomes increasingly localized at the interface, with most of the field contained on the Ag side. Figure 5(c) illustrates the energy density within the RPP mode at $3.98 \mathrm{eV}\left(\lambda_{\mathrm{vac}}\right.$ $=312 \mathrm{~nm}$ ), just beyond the onset of the RPP. This region of the RPP mode is marked by a damped oscillation into the dielectric and an evanescent wave into the metal. For increasing energy [Fig. 5(d), $\left.6.17 \mathrm{eV}, \lambda_{\mathrm{vac}}=201 \mathrm{~nm}\right]$, the maximal amplitude of each wave decreases and oscillations become increasingly damped. This behavior is in contrast to the radiative mode predicted by the FEG model, characterized by a pure photon mode in the dielectric with a constant energy density of $u \sim 1 \mathrm{~J} / \mathrm{m}^{3}$ in the metal and $u \sim 21 \mathrm{~J} / \mathrm{m}^{3}$ in $\mathrm{SiO}_{2}$ (data not shown).

While study of decoupled plasmons provides a first-order approximation to SPP behavior in bulk metals, the results of Fig. 6 suggest that this approximation breaks down for metal films no thicker than approximately $70 \mathrm{~nm}$. As the thickness of the film is decreased, one can no longer ignore the interaction between surface plasmons on each side of the metal film.

\section{THIN Ag FILMS: COUPLED SURFACE PLASMONS}

The first theoretical study of thin-film surface plasmons may be attributed to Kliewer and Fuchs, ${ }^{34-36}$ who determined 
the long-wavelength collective polariton excitations of an ionic crystal. In their work, the dispersion relations were determined by imposing the appropriate boundary conditions on Maxwell's equations for an isotropic metallic slab. An alternate derivation based on Fresnel's equations has been suggested by Raether, ${ }^{22}$ but the results are equivalent. A schematic of the geometry considered is shown in Fig. 6. Assuming a metal film of thickness $d$ centered at $z=0$ and embedded in identical dielectric media described by $\varepsilon_{2}$, the dispersion relations take the form

$$
\begin{aligned}
& L+: \varepsilon_{1} k_{z 2}+\varepsilon_{2} k_{z 1} \tanh \left(\frac{-i k_{z 1} d}{2}\right)=0, \\
& L-: \varepsilon_{1} k_{z 2}+\varepsilon_{2} k_{z 1} \operatorname{coth}\left(\frac{-i k_{z 1} d}{2}\right)=0
\end{aligned}
$$

with $k_{z 1}$ and $k_{z 2}$ defined by Eq. (4). Accordingly, each solution represents a distinct coupling mode between the interface plasmons and reflects the symmetry of the electric field. As seen in Fig. 6(a), the field of the higher energy mode $(L+)$ is antisymmetric with respect to the $z=0$ plane; the converse is true of the lower energy, symmetric mode $(L+)$, shown in Fig. 6(b). ${ }^{37}$

To obtain the electric field, Maxwell's equations must be solved under constraint of tangential electric field and normal displacement field continuity. The solutions in all spatial regions may be written as

$$
E(x, z, t)=\left(E_{x} \hat{x}+E_{y} \hat{y}+E_{z} \hat{z}\right) e^{i\left(k_{x} x-\omega t\right)}
$$

with field components inside the slab given by

$$
\begin{gathered}
E_{x}^{\text {metal }}=e^{-i k_{z 1} z} \pm e^{i k_{z 1} z}, \\
E_{y}^{\text {metal }}=\left\{\begin{array}{c}
\cos \left(k_{z 1} z\right), \\
\sin \left(-k_{z 1} z\right),
\end{array}\right. \\
E_{z}^{\text {metal }}=\left(\frac{k_{x}}{k_{z 1}}\right)\left(e^{-i k_{z 1} z} \pm e^{i k_{z 1} z}\right)
\end{gathered}
$$

and field components outside the slab assuming the form

$$
\begin{gathered}
E_{x}^{\text {dielectric }}=\left(e^{-i k_{z 1} d / 2} \pm e^{i k_{z 1} d / 2}\right) e^{i k_{z 2}(z-d / 2),} \\
E_{y}^{\text {dielectric }}=\left\{\begin{array}{l}
\cos \left(k_{z 1} d / 2\right) e^{i k_{z 2}(z-d / 2)}, \\
\sin \left(k_{z 1} d / 2\right) e^{i k_{z 2}(z-d / 2)},
\end{array}\right. \\
E_{z}^{\text {dielectric }}=\left(\frac{\varepsilon_{1} k_{x}}{\varepsilon_{2} k_{z 1}}\right)\left(e^{-i k_{z 1} d / 2} \pm e^{i k_{z 1} d / 2}\right) e^{i k_{z 2}(z-d / 2)} .
\end{gathered}
$$

Accordingly, the upper sign convention represents the solution to plasmon excitations in the symmetric field configuration $(L-)$, while the lower sign convention represents solutions for the antisymmetric field configuration $(L+)$.

While no exact solution exists for the dispersion relations (8) and (9) in the real frequency domain, $|L+|$ and $|L-|$ do appear in the denominator of the expression for the energy loss probability of electrons penetrating a thin metal film..$^{11,38}$ It follows that minimization of $|L+|$ and $|L-|$ corresponds to
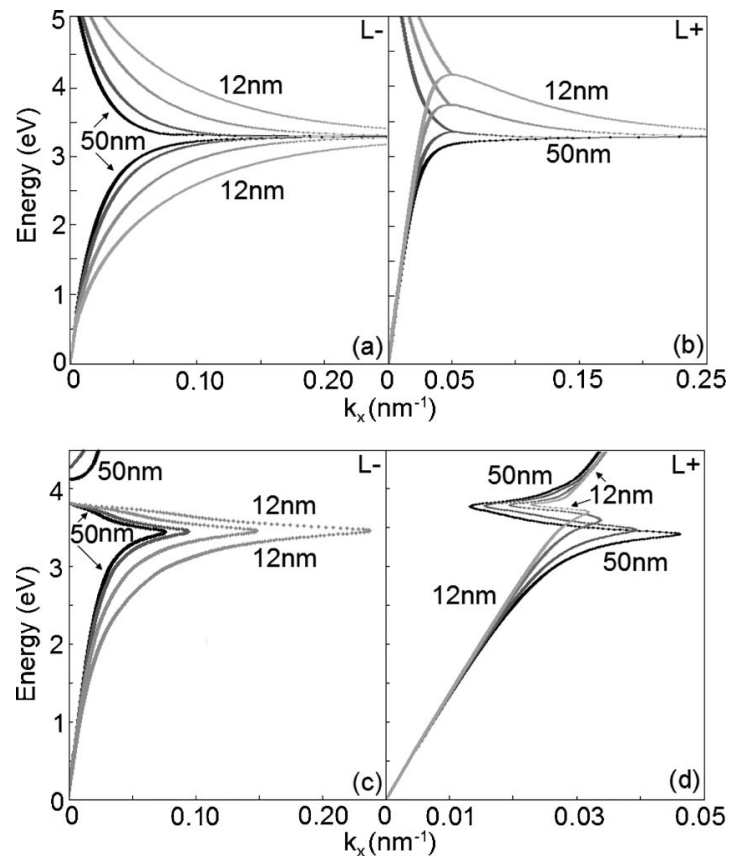

FIG. 7. Dispersion for the $\mathrm{SiO}_{2} / \mathrm{Ag} / \mathrm{SiO}_{2}$ geometry for four different Ag thicknesses (12, 20, 35, and $50 \mathrm{~nm})$. Dispersion computed using the free electron gas dielectric function is plotted in panels (a) and (b) while dispersion computed using optical constants of Johnson and Christy is shown in (c) and (d). (a), (c) Lower energy, symmetric mode $(L-)$, (b), (d) Higher energy, antisymmetric mode $(L+)$.

a maximum in the energy loss probability and hence a maximum in the plasmon intensity distribution.

To obtain the complex wave vectors, numerical solution of $\min |L+|$ and $\min |L-|$ was accomplished through implementation of a two-dimensional unconstrained Nelder-Mead minimization algorithm. The routine relied on an adaptive simplex method ${ }^{39}$ and guaranteed convergence through the strict convexity of $|L+|$ and $|L-|$. All returned wave vectors minimized the functions $|L+|$ and $|L-|$ with a tolerance of $10^{-13} \mathrm{~nm}^{-1}$.

Figures 7(a) and 7(b) plot the thin-film dispersion relations obtained via the adaptive simplex method, considering $\mathrm{Ag}$ as a free electron gas with bulk plasma frequency $\omega_{p}$ $=8.85 \times 10^{15} \mathrm{~s}^{-1}$. The results are in good agreement with the theoretical dispersion characteristics predicted by Kliewer and Fuchs. $^{2}$ As film thickness is decreased from 50 to $12 \mathrm{~nm}$, the degeneracy of the two interface plasmons is split, with each mode asymptoting at short wave vectors to the light line and at large wave vectors to the free electron gas SP resonant frequency $\omega_{\mathrm{SP}}=\omega_{p} /\left[1+\varepsilon_{2}\right]^{1 / 2}$.

Figure 7(a) plots the $L$ - mode dispersion for a free electron gas, revealing a significant enhancement in plasmon wave vector for decreasing film thickness at energies well below the SP resonance. Exploiting these modes in thin films, extremely short plasmon wavelengths can be achieved at wavelengths well above that of the plasmon resonance. Figure 7(b) plots the free electron gas $L+$ mode dispersion, exhibiting enhanced maxima at energies above $\omega_{\mathrm{SP}}$ for decreasing film thicknesses. Approaching the SP resonant fre- 
quency from above, a negative group velocity is observed for this mode for larger wave vectors. In both figures, we see that the model also returns solutions above the typical SP free electron gas dispersion curves, in direct analogy with the forbidden modes of the thick film limit. In both the symmetric and antisymmetric charge configurations, these highly damped, short-lived modes join the SP dispersion curve at the point of zero slope, indicating the lower energy bound of the classical plasmon bandgap.

Figures 7(c) and 7(d) plot the dispersion curves generated using the Ag optical constants of Johnson and Christy. Nearly identical results were obtained using the optical constants of Palik. As with the semi-infinite thick film geometry, the dispersion relations reveal the presence of SPP, RPP, and quasibound modes. Though not shown in this figure, dispersion of a $100 \mathrm{~nm} \mathrm{Ag}$ film behaves just as in the decoupled SP geometry (see Fig. 2).

For the antisymmetric plasmon mode $[L-$, Fig. $7(\mathrm{c})]$, the JC thin-film SPP exhibits behavior qualitatively similar to the FEG SPP for energies below the plasmon resonance. As seen, at a given frequency, the thin-film SPP is pushed toward higher wave vectors as film thickness is decreased. However, unlike FEG dispersion, thin-film SPPs exhibit a maximum wave vector at $\omega_{\mathrm{SP}}$ rather than asymptoting to this frequency. For a 12-nm-thick film, the SPP has a wave vector as large as $k_{x} \sim 0.2 \mathrm{~nm}^{-1}$ at $\omega_{\mathrm{SP}}$, corresponding to a surface plasmon wavelength of $2 \pi / k_{x}=30 \mathrm{~nm}$. Above $\omega_{\mathrm{SP}}$, the quasibound modes are observed up to $3.8 \mathrm{eV}$, marking the onset of the RPP. Note that the RPP of the symmetric mode is found at increasingly higher energies as film thickness is decreased. This behavior indicates that, despite the existence of quasibound modes, thin films in the symmetric mode still exhibit a stop band between SPP and RPP dispersion branches.

For the asymmetric plasmon mode $[L+$, Fig. $7(d)]$, the thin-film SPP dispersion shifts toward smaller wave vectors at lower energies as film thickness decreases (just as in the free electron gas model of Fig. 7(b)). As energy is increased above the plasmon resonance, the antisymmetric SPP reaches a maximum wave vector before cycling back to smaller wave vectors through the quasibound modes and into the RPP. Unlike the free electron gas dispersion relation, the thin-film antisymmetric SPP does not asymptote to $\omega_{\mathrm{SP}}$ from above. In fact, for energies up through the SP resonance, the $L+$ dispersion relation for the $12-\mathrm{nm}$ film is almost indistinguishable from the light line. The observed behavior is quite unlike that predicted by the free electron model, but seems to be in accord with dispersion curves from experimental electron loss spectra (Ref. 10).

Figure 8 illustrates surface plasmon propagation length for both the symmetric and antisymmetric modes as a function of wavelength, using the optical constants from the Palik Handbook. (Propagation using the optical constants of Johnson and Christy is qualitatively similar.) As this plot reveals, as film thickness is decreased, propagation is reduced to the micron scale for the symmetric mode $(L-)$ and increased to tens of centimeters for the asymmetric mode $(L+)$. While a discrepancy of about a factor of 10 is observed between predicted propagation length using the data of Johnson and Christy and Palik, long-range propagation is

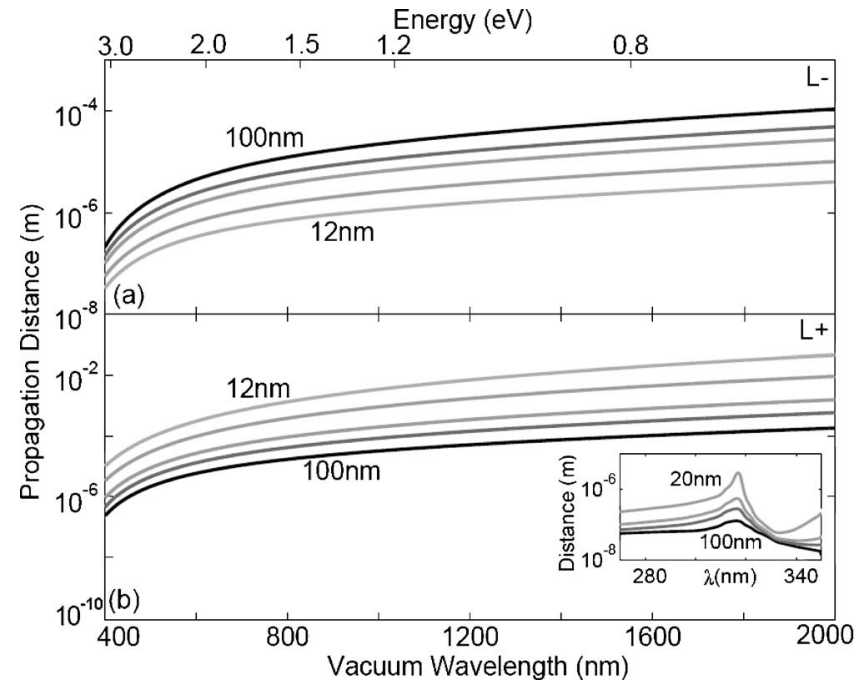

FIG. 8. $\mathrm{SiO}_{2} / \mathrm{Ag} / \mathrm{SiO}_{2}$ plasmon propagation lengths computed with the optical constants from Palik calculated for five different $\mathrm{Ag}$ thicknesses $(12,20,35,50$, and $100 \mathrm{~nm})$. (a) Lower energy, symmetric field mode $(L-)$, (b) higher energy, antisymmetric field mode $(L+)$. The inset of (b) plots propagation distance for wavelengths characteristic of the regime of anamolous Ag dispersion.

still obtained in both cases. For a 12-nm-thick film, propagation distances for the symmetric mode approach $18 \mu \mathrm{m}$ $(2 \mu \mathrm{m})(\mathrm{JC} /$ Palik data) at a free-space wavelength $1550 \mathrm{~nm}$. At the same wavelength, propagation distances for the antisymmetric mode are calculated to be as large as $7 \mathrm{~cm}$ $(1 \mathrm{~cm})$.

As in the decoupled SP geometry, the propagation distance in thin films exhibits a minimum about the surface plasmon resonance and a local maximum about the region of anomalous dispersion [see inset, Fig. 8(b)]. Note that for the $L+$ mode, the minimum in propagation shifts to shorter wavelengths as thickness is decreased, in agreement with the energy shift of the wave vector maximum observed in Fig. $7(\mathrm{~d})$. For thicker films $(d>50 \mathrm{~nm})$ in the antisymmetric field configuration, this maximum does not much exceed the amplitude of the local maximum of the semi-infinite geometry observed in Fig. 3. However, as film thickness is decreased below $50 \mathrm{~nm}$, propagation appears to approach the micron scale. Such results do not seem to be a numerical artifact for the $L+$ mode and thus may be a promising avenue for future subwavelength waveguiding research, provided signal dispersion is not dominant. To the contrary, enhanced propagation at short wavelengths could not be observed in the $L-$ mode, as the corresponding wavelength regime represents the transition from the stop-band regime (where $k_{x}=0$ ) to RPP modes.

While experiments used to measure propagation distances are typically performed by critical coupling of light at a particular free space frequency, waveguiding applications require the simultaneous optimization of surface plasmon wavelength and propagation distance. Figure 9 illustrates the dependence of propagation length on SP wave vector for bound surface plasmon polariton modes. As seen in Fig. 9(a), plasmon propagation distance for the symmetric mode $(L-)$ is a global maximum for thick films with shorter SP 


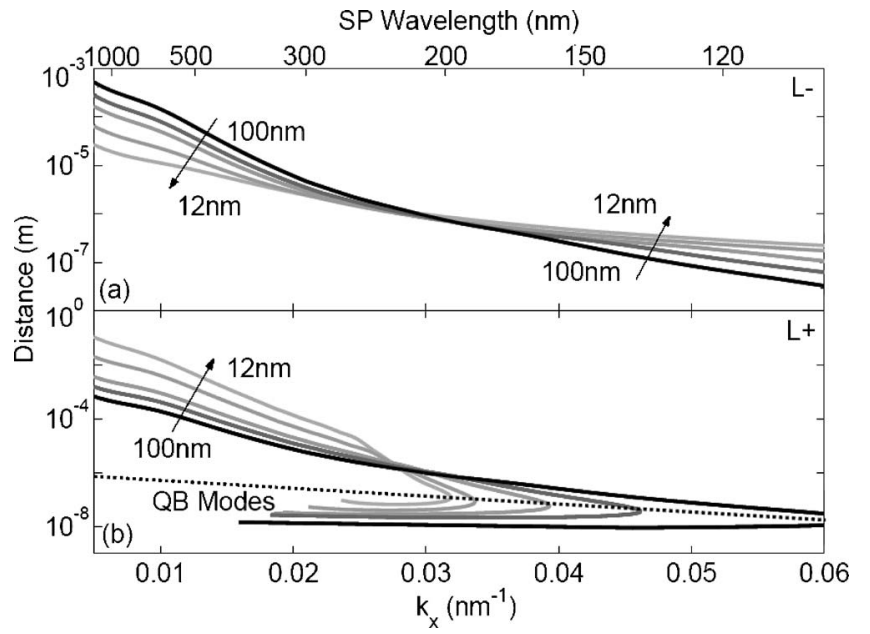

FIG. 9. Propagation distance as a function of in-plane wave vector for five different $\mathrm{Ag}$ film thicknesses (12, 20, 35, 50, and $100 \mathrm{~nm})$. Arrows indicate the trend of decreasing film thickness. For both the symmetric field mode $[L-,(\mathrm{a})]$ and the antisymmetric field mode $[L+,(b)]$, the optimal combination of low damping and long wave vector is achieved at $\lambda_{\mathrm{SP}}=210 \mathrm{~nm}$.

wave vectors (larger SP wavelengths). However, for SP wave vectors greater than $k_{x}=0.03 \mathrm{~nm}^{-1}\left(\lambda_{\mathrm{SP}}<210 \mathrm{~nm}\right)$, longer propagation distances are observed for thinner films. A 12-nm-thick Ag film, for example, approaches propagation lengths of approximately $0.3 \mu \mathrm{m}$ at an SP wave vector of $0.05 \mathrm{~nm}^{-1}\left(\lambda_{\mathrm{SP}}=125 \mathrm{~nm}\right)$.

In contrast, plasmon propagation distance for the antisymmetric mode $(L+)$ is a global maximum for thin films with shorter SP wave vectors (larger SP wavelengths). However, as SP wavelengths are decreased beyond $\lambda_{\mathrm{SP}} \sim 210 \mathrm{~nm}$, thicker films optimize the tradeoffs between plasmon localization and loss. While a 12-nm-thick film can achieve propagation distances of order $2 \mathrm{~cm}$ at an SP wave vector of $0.01 \mathrm{~nm}^{-1} \quad\left(\lambda_{\mathrm{SP}}=630 \mathrm{~nm}\right)$, propagation quickly falls to $370 \mathrm{~nm}$ at an SP wave vector of $0.03 \mathrm{~nm}^{-1}\left(\lambda_{\mathrm{SP}}<210 \mathrm{~nm}\right)$.

It is noteworthy to observe that the intersection of all curves at $k_{x}=0.03 \mathrm{~nm}^{-1}$ does not occur at the transition between SPP and QB modes. Rather, this crossover occurs at energies well below the SP resonance, in regions where the group velocity is still non-negligible. Mathematically, this node represents the compromise between signal speed (as determined by the real component of the wave vector) and plasmon damping (as determined by the imaginary component). The physical origin of the node at $k_{x}=0.03 \mathrm{~nm}^{-1}$ remains under consideration, but may be related to a crossover in the relative strength of transverse and longitudinal SP fields. Just as observed in the semi-infinite geometry, then, large surface plasmon wave vectors are achieved at the expense of propagation length. However, maximum mode propagation for a given SP wave vector can be obtained by selecting films of an optimal thickness.

Figure 10 plots the 1/e decay length (skin depth) of the surface plasmon electric field into $\mathrm{SiO}_{2}$ as a function of wavelength for both the symmetric and antisymmetric modes, calculated using Johnson and Christy optical constants. For oscillatory mode profiles, 1/e decay length was

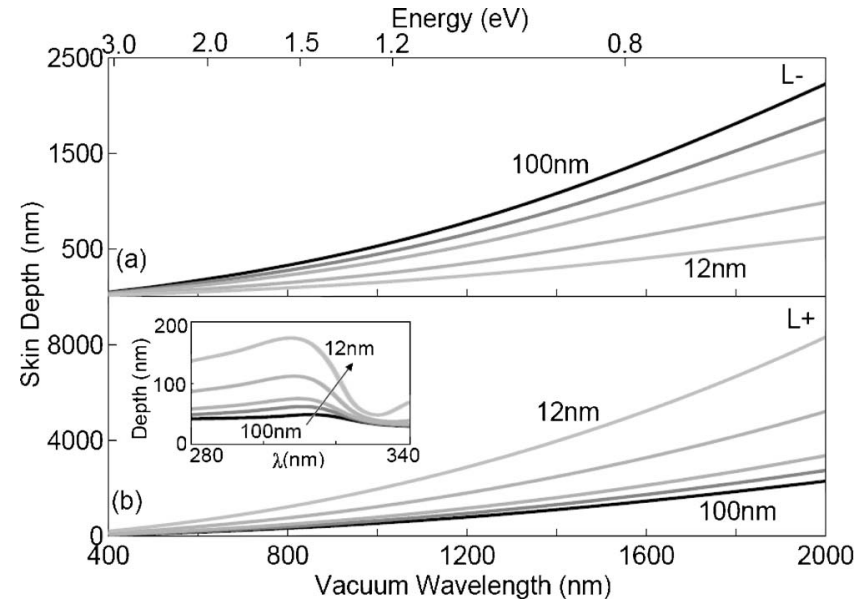

FIG. 10. Skin depth of the $\mathrm{SiO}_{2} / \mathrm{Ag} / \mathrm{SiO}_{2}$ bound surface plasmon, computed using the optical constants of Johnson and Christy. (a) Lower energy, symmetric mode $(L-)$, (b) higher energy, antisymmetric mode $(L+)$ for five different film thicknesses $(12,20,35$, 50 , and $100 \mathrm{~nm}$ ). The inset shows field penetration depth into the dielectric for wavelengths below the surface plasmon resonant wavelength, with the arrow indicating the trend of decreasing waveguide thickness.

determined by considering decay of the exponential envelope. As can be seen, skin depth of the bound antisymmetric mode increases into the dielectric with decreasing film thickness. Conversely, for the bound symmetric mode, field penetration into the dielectric decreases with decreasing film thickness. At near-infrared wavelengths $(1550 \mathrm{~nm})$, the electric field of a 12-nm-thick film in the antisymmetric charge configuration is highly delocalized, extending to $5 \mu \mathrm{m}$ in the $\mathrm{SiO}_{2}$. For the symmetric charge configuration, however, the field only extends $\sim 400 \mathrm{~nm}$ into the dielectric for the same film thickness at the same wavelength.

As in infinitely thick films (see Fig. 4), the skin depth of the antisymmetric $(L+)$ quasibound and radiative plasmons exhibits a local maxima for energies above the plasmon resonance (see Fig. 10, inset). The magnitude of this maxima increases with decreasing film thickness, more than doubling for each film thickness down to $12 \mathrm{~nm}$. Recalling the local maximum in the $L+$ propagation length plot [Fig. 8(b)], this increase in skin depth does not appear to be an artifact for the $L+$ mode. Assuming an electrostatic limit, this phenomenon could result from disentanglement of the plasmon-polariton via interaction of bound surface charge.

To better understand the relation between field symmetry and propagation length, it is useful to consider the localization of energy density within the waveguide. Figure 11 illustrates the electric energy density distribution as a function of distance from the metal-dielectric interface for the $L-$ and $L+$ modes, respectively. Field distributions in $\mathrm{Ag}$ and $\mathrm{SiO}_{2}$ are plotted separately in panels (a),(c) and (b),(d), and Ag film thickness is varied between 12 and $100 \mathrm{~nm}$. (Note that the energy density for the 100-nm-thick film has been scaled by a factor of $\frac{1}{4}$ and that in the (b),(d) panels, the metaldielectric interface has been normalized to $z=0$ for all film thicknesses.) The energy is fixed at $2.75 \mathrm{eV}\left(\lambda_{\mathrm{vac}}=450 \mathrm{~nm}\right.$, i.e., a wavelength regime of maximum dispersion), and the 

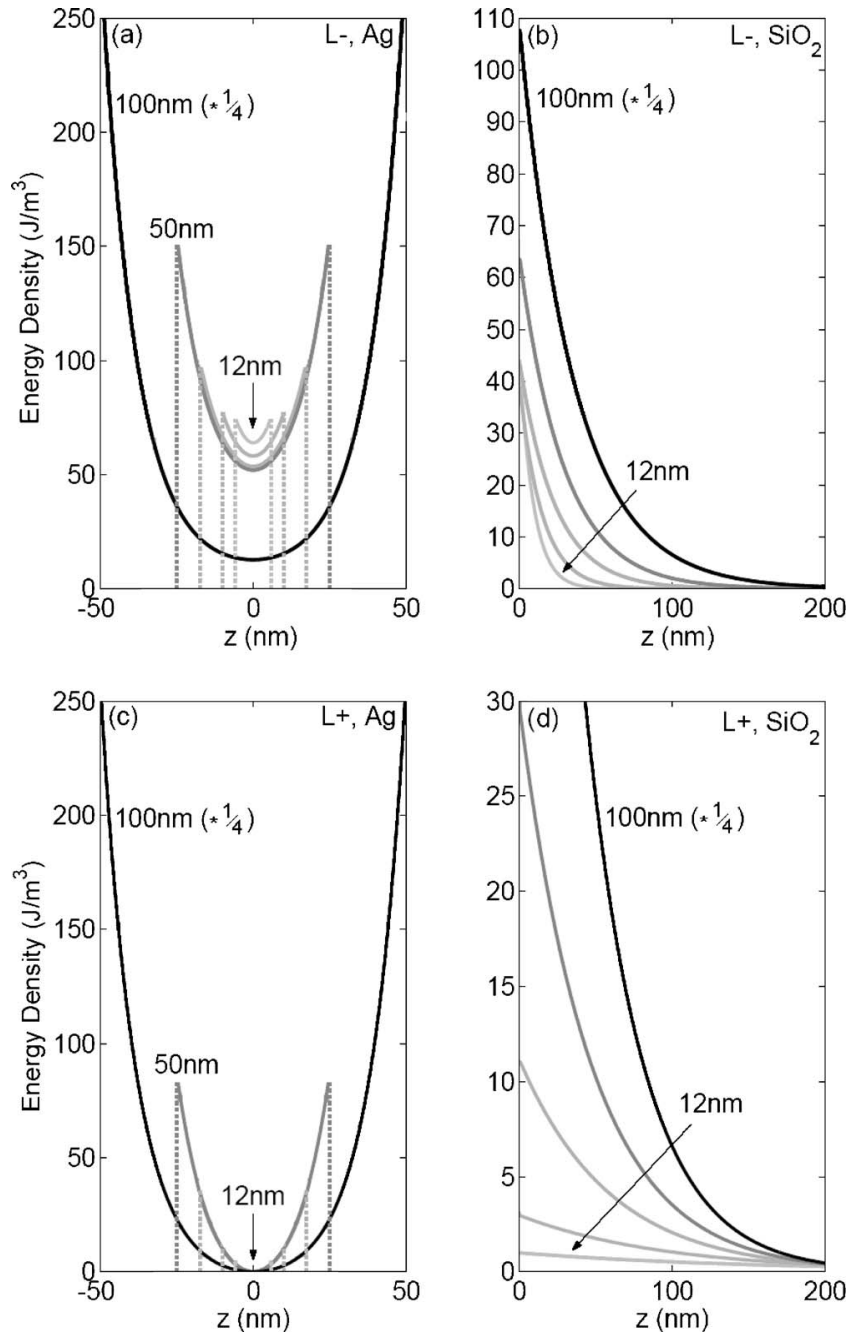

FIG. 11. Spatial distribution of energy density for the $\mathrm{SiO}_{2} / \mathrm{Ag} / \mathrm{SiO}_{2}$ slab geometry plotted in the metal (a),(c) and dielectric (b),(d) for both the symmetric field mode (a),(b) and the antisymmetric mode (c),(d) using the optical constants of Johnson and Christy. Ag film thicknesses shown are varied from $100 \mathrm{~nm}$ down to $12 \mathrm{~nm}$, with arrows indicating the trend of decreasing thickness. The free space wavelength is set to $\lambda=450 \mathrm{~nm}$, a region of maximum bound surface plasmon dispersion.

incident electric field is normalized to $1 N / C$. As seen in the figure, the energy density within the metal is maximized at the metal-dielectric interface $(z= \pm d / 2)$ and decreases with near quadratic dependence into the waveguide.

For the $L$ mode, energy density at the $\mathrm{Ag} / \mathrm{SiO}_{2}$ interface decreases with decreasing thickness, reaching a density of $u=75 \mathrm{~J} / \mathrm{m}^{3}$ at the metal surface for a 12-nm-thick film [Fig. 11(a)]. Within the metal, energy density remains nonnegligible as film thickness is decreased, with thinner films exhibiting higher energy densities at the waveguide center than thicker films. Energy density within a 12-nm-thick Ag film, for instance, is minimized at $u \sim 65 \mathrm{~J} / \mathrm{m}^{3}$, while the minimum energy density within a 100 -nm-thick film is $48 \mathrm{~J} / \mathrm{m}^{3}$. In contrast, for the antisymmetric mode $[L+$, Fig. $11(\mathrm{c})]$, energy density at the $\mathrm{Ag} / \mathrm{SiO}_{2}$ interface decreases with decreasing film thickness and exhibits a node at $z=0$. The maximum energy density at the $\mathrm{Ag} / \mathrm{SiO}_{2}$ interface for a
$12 \mathrm{~nm}$ film is approximately $3 \mathrm{~J} / \mathrm{m}^{3}$, a $99.7 \%$ decrease from the maximal energy density observed for a $100 \mathrm{~nm} \mathrm{Ag} \mathrm{film.}$

Outside the metal, the energy density generally decreases with decreasing film thickness for both the symmetric and antisymmetric modes [Figs. 11(b) and 11(d)]. As seen in Fig. 11(b), energy densities for films in the symmetric SP field configuration fall fairly quickly: for example, density of a 12-nm-thick film decays to $1 / e$ of its maximal amplitude within $10 \mathrm{~nm}$. For the antisymmetric mode, however, the energy density extends well into the dielectric, with $1 / e$ lengths exceeding $180 \mathrm{~nm}$ for a $12 \mathrm{~nm} \mathrm{Ag} \mathrm{film} \mathrm{[Fig.} \mathrm{11(d)].} \mathrm{At} \mathrm{the}$ metal/dielectric interface, energy densities in the dielectric are typically less than half the maximum value observed in the metal. Interestingly, energy density magnitudes are significantly lower at the metallodielectric interface for the antisymmetric field configuration, reflecting the lower losses observed for such modes.

\section{CONCLUSIONS}

The potential for surface plasmons to realize long-range waveguiding in the infrared has been known for nearly thirty years. However, their optoelectronic applications have not been realized until recently. No doubt this conversion is due to the remarkable progress in nanoscale fabrication, allowing structures to be designed and studied on scales where the classical meets the quantum mechanical. As experiments become more sophisticated and relevant to technological applications, it becomes crucial to understand-quantitativelythe dispersion, localization, and attenuation characteristics of surface plasmons over a wide range of wavelengths. To first order, this work has provided such an analysis.

The preceding sections have served to outline the properties of Ag slab plasmons, considering the metal as described by empirically determined optical constants rather than a free electron gas model. Using the data sets of Johnson and Christy and from the Palik Handbook, surface plasmon dispersion, propagation, skin depth, and energy density were analyzed for both thick and thin films. Results were obtained over the wavelength range from 200 to $2000 \mathrm{~nm}$ and for all modes supported by the structure.

In thick films, the dispersion curve was found to exhibit three distinct branches corresponding to surface plasmonpolariton, radiative plasmon-polariton, and a feature not previously reported, which we term quasibound modes. Propagation distances of these modes approached the micron scale in the near infrared and subnanometer scales at the SPP resonant frequency. For higher frequencies, the propagation distance exhibited a local maximum, corresponding to the transition between the QB and RPP modes. Interestingly, at this wavelength, electric field penetration into the metal reached a maximum, while at the SPP resonance field penetration approached single nanometer scales. These results suggested that high field localization alone is not necessarily detrimental to plasmon propagation. Moreover, calculations using dielectrics other than $\mathrm{SiO}_{2}$ indicated the potential for dynamic tuning of propagation length. By altering, for instance, the refractive index of a material, propagation can be switched distances of several microns, provided $\Delta n \geqslant 0.1$. 
The properties of thin-film plasmons were obtained by implementing a numerical algorithm to determine the maxima of the electron energy loss spectra. Considering Ag as a free electron gas, results seemed to be in strong accord with previous theoretical predictions. However, the dispersion characteristics obtained considering the empirical optical constants of Ag deviated significantly from the free electron gas data. Most notably, the dispersion curve terminated on resonance at a finite wave vector, exhibited quasibound modes and, in the antisymmetric field configuration (symmetric charge configuration), did not asymptote to $\omega_{\mathrm{SP}}$ from above.

The propagation characteristics of all thin-film modes were determined, and results were justified by considering field penetration and energy density decay in the metal and dielectric. Of the two modes $(L+$ and $L-)$, it was found that the symmetric $(L-)$ mode surface plasmon exhibited decreasing propagation length and enhanced field confinement in the metal with decreasing film thickness. In contrast, the antisymmetric $(L+)$ mode plasmon exhibited increasing propagation length and decreasing plasmon wave vector with decreasing film thickness. Propagation distances generally decreased with increasing surface plasmon momentum for both the $L-$ and $L+$ modes. Interesting, identical symmetric $(L-)$ and antisymmetric $(L+)$ plasmon propagation lengths were observed for all film thicknesses at an SP wave vector of $k_{x} \sim 0.03 \mathrm{~nm}^{-1}$. While long-range propagation in the infrared was accompanied by a broad energy density distribution in the dielectric, non-negligible propagation distances were observed over a narrow bandwidth at ultraviolet wave- lengths. However, more detailed dispersion analysis is required to determine if such long-range surface plasmons are accessible and observable.

As the above results suggest, thin film plasmons excited from the ultraviolet to the near-infrared may find applications in microphotonic and nanophotonic waveguiding and switching. Current experimental techniques, supplemented by the numerical analysis presented, will facilitate progress towards such novel plasmonic venues.

\section{ACKNOWLEDGMENTS}

This work has benefited from numerous discussions with friends and colleagues, most particularly Jeroen Kalkman, Hans Mertens, Nhat Vu, and Adriaan Tip. Financial support at Caltech was provided by the Air Force Office of Scientific Research, MURI Grant No. FA9550-04-1-0434. One of us (J.A.D.) acknowledges financial support from the National Science Foundation and the Department of Defense Army Research Office. Work at AMOLF is part of the research program of FOM and is financially supported by NWO. A.P. gratefully acknowledges the hospitality of the Atwater group during his sabbatical stay.

\section{APPENDIX: FUNCTIONAL FITS TO EMPIRICAL OPTICAL CONSTANTS}

Ag dielectric constant $\left(\varepsilon=\varepsilon_{1}{ }^{\prime}+\varepsilon_{1}{ }^{\prime \prime}\right)$ fitting parameters, Johnson and Christy data:

$$
\begin{gathered}
\varepsilon_{1}{ }^{\prime}= \begin{cases}\text { cubic spline, } & \lambda<500 \mathrm{~nm}, \\
29.34-0.11028 \lambda+\left(1.1218 \times 10^{-4}\right) \lambda^{2}-\left(1.08164 \times 10^{-7}\right) \lambda^{3}+\left(2.44496 \times 10^{-11}\right) \lambda^{4}, & \lambda \geqslant 500 \mathrm{~nm},\end{cases} \\
\varepsilon_{1}{ }^{\prime}= \begin{cases}\text { cubic spline, } & \lambda<400 \mathrm{~nm}, \\
-1.753+0.009962 \lambda-\left(1.696 \times 10^{-5}\right) \lambda^{2}+\left(1.178 \times 10^{-8}\right) \lambda^{3}-\left(2.334 \times 10^{-12}\right) \lambda^{4}, & \lambda \geqslant 400 \mathrm{~nm} .\end{cases}
\end{gathered}
$$

Ag dielectric constant $\left(\varepsilon=\varepsilon_{1}{ }^{\prime}+\varepsilon_{1}{ }^{\prime \prime}\right)$ fitting parameters, Palik data:

$$
\begin{gathered}
\varepsilon_{1}{ }^{\prime}= \begin{cases}\text { cubic spline, } & \lambda<350 \mathrm{~nm}, \\
10.314-0.026295 \lambda-\left(2.51 \times 10^{-5}\right) \lambda^{2}, & \lambda \geqslant 350 \mathrm{~nm},\end{cases} \\
\varepsilon_{1}{ }^{\prime \prime}= \begin{cases}\text { cubic spline, } & \lambda<350 \mathrm{~nm}, \\
1.0481-0.003259 \lambda+\left(5.3387 \times 10^{-6}\right) \lambda^{2}, & \lambda \geqslant 350 \mathrm{~nm}\end{cases}
\end{gathered}
$$

$\mathrm{SiO}_{2}$ dielectric constant fitting parameters, Palik data:

$$
\varepsilon_{2}= \begin{cases}2.806-0.00347 \lambda+\left(6.10502 \times 10^{-6}\right) \lambda^{2}-\left(3.68627 \times 10^{-9}\right) \lambda^{3}, & \lambda<612 \mathrm{~nm}, \\ 2.222-\left(2.46178 \times 10^{-4}\right) \lambda+\left(1.71928 \times 10^{-7}\right) \lambda^{2}-\left(4.49923 \times 10^{-11}\right) \lambda^{3}, & \lambda \geqslant 612 \mathrm{~nm} .\end{cases}
$$

\footnotetext{
*Electronic address: jdionne@ caltech.edu

${ }^{1}$ R. H. Ritchie, Phys. Rev. 106, 874 (1957).
}

\footnotetext{
${ }^{2}$ K. L. Kliewer and R. Fuchs, Phys. Rev. 153, 498 (1967).

${ }^{3}$ E. N. Economou, Phys. Rev. 182, 539 (1969).
} 
${ }^{4}$ J. J. Burke, G. I. Stegeman, and T. Tamir, Phys. Rev. B 33, 5186 (1985).

${ }^{5}$ U. Fano, Phys. Rev. 118, 451 (1960).

${ }^{6}$ J. C. Weeber, J. R. Krenn, A. Dereux, B. Lamprecht, Y. Lacroute, and J. P. Goudonnet, Phys. Rev. B 64, 045411 (2001).

${ }^{7}$ A. Zayats and I. Smolyaninov, J. Opt. A, Pure Appl. Opt. 5, S16 (2003).

${ }^{8}$ P. Dawson, F. de Fornel, and J. P. Goudonnet, Phys. Rev. Lett. 72, 2927 (1994).

${ }^{9}$ F. Silly, A. O. Gusev, A. Taleb, F. Charra, and M-P. Pileni, Phys. Rev. Lett. 84, 5840 (2000)

${ }^{10}$ R. Vincent and J. Silcox, Phys. Rev. Lett. 31, 1487 (1973).

${ }^{11}$ R. B. Pettit, J. Silcox, and R. Vincent, Phys. Rev. B 11, 3116 (1975).

${ }^{12}$ T. Velinov, M. G. Somekh, and S. Liu, Appl. Phys. Lett. 75, 3908 (1999).

${ }^{13}$ B. Lamprecht, J. R. Krenn, G. Schider, H. Ditlbacher, M. Salerno, N. Felidj, A. Leitner, F. R. Aussenegg, and J. C. Weeber, Appl. Phys. Lett. 79, 51 (2001).

${ }^{14}$ T. Nikolajsen, K. Leosson, I. Salakhutdinv, and S. I. Bozhevolnyi, Appl. Phys. Lett. 82, 668 (2003).

${ }^{15}$ P. Berini, Opt. Lett. 24, 1011 (1999).

${ }^{16}$ P. Berini, Phys. Rev. B 61, 10484 (2000).

${ }^{17}$ P. Berini, Opt. Express 7, 329 (2000).

${ }^{18}$ P. Berini, Phys. Rev. B 63, 125417 (2001).

${ }^{19}$ P. B. Johnson and R. W. Christy, Phys. Rev. B 6, 4370 (1972).

${ }^{20}$ Handbook of Optical Constants of Solids, edited by E. Palik (Academic Press, New York, 1985).

${ }^{21}$ E. Palik and G. Ghosh, Handbook of Optical Constants of Solids II (Academic Press, New York, 1991).

${ }^{22}$ H. Raether, Surface Plasmons on Smooth and Rough Surfaces and on Gratings (Springer-Verlag, Berlin, 1988).

${ }^{23}$ E. A. Stern and R. A. Ferrell, Phys. Rev. 120, 130 (1960).

${ }^{24}$ J. B. Pendry, L. Martin-Moreno, and F. J. Garcia-Vidal, Science
35, 847 (2004).

${ }^{25}$ G. Leveque, C. G. Olson, and D. W. Lynch, Phys. Rev. B 27, 4654 (1983).

${ }^{26}$ P. Winsemius, F. F. van Kampen, H. P. Lengkeek, and C. G. van Went, J. Phys. F: Met. Phys. 6, 1583 (1976).

${ }^{27}$ B. Dold and R. Mecke, Optik (Stuttgart) 22, 435 (1965).

${ }^{28}$ For noble metals, $\omega_{p}$ is often in the optical frequency regime between $10^{15}$ and $10^{16} \mathrm{~s}^{-1}$; see, for instance, S. S. Martinos, Phys. Rev. B 40, 8558 (1989) or reference 19.

${ }^{29}$ An analogous termination at finite $k_{x}$ can be observed in FEG dispersion by including a finite scattering rate $\gamma$ in Eq. (1).

${ }^{30}$ M. Tanaka, Plasma Phys. Controlled Fusion 31, 1049 (1989).

${ }^{31}$ Damping in $\mathrm{Si}$ was accounted for in the usual way, via inclusion of the imaginary component of the dielectric function.

${ }^{32}$ While typical refractive index differentials in semiconductors do not exceed $1 \%$, nonlinear index changes as high as $\Delta n=0.14$ have been observed in multilayer quantum wells. See, for instance, L. Brzozowski, E. Sargent, A. S. Thorpe, and M. Extavour, Appl. Phys. Lett. 82, 4429 (2003).

${ }^{33}$ L. D. Landau and E. M. Lifshitz, Electrodynamics of Continuous Media, 2nd ed. (Butterworth-Heinenann, Oxford, 1984).

${ }^{34}$ K. L. Kliewer and R. Fuchs, Phys. Rev. 140, A2076 (1965).

${ }^{35}$ K. L. Kliewer and R. Fuchs, Phys. Rev. 144, 495 (1966).

${ }^{36}$ K. L. Kliewer and R. Fuchs, Phys. Rev. 150, 573 (1966).

${ }^{37}$ In describing the symmetry of the field, we address the symmetry with respect to a longitudinal slice through the waveguide in the direction of plasmon propagation. Because charges of opposite sign lie directly across from each other in the symmetric field $(L-)$ configuration, this mode corresponds to an antisymmetric charge distribution. Likewise, the antisymmetric field $(L+)$ mode corresponds to a symmetric charge distribution.

${ }^{38}$ E. Kröger, Z. Phys. 216, 115 (1968).

${ }^{39}$ J. A. Nelder and R. Mead, Comput. J. 7, 308 (1965). 